\title{
Effective Prestress Force Estimation in Prestress Concrete Beam with Dynamical Method
}

\author{
Lanlin ZOU ${ }^{\mathrm{a}}$, Yuan YAN $^{\mathrm{b}}$, Xinglin $\mathrm{ZHOU}^{\mathrm{c}}$ \\ School of Automobile and Traffic Engineering, Wuhan University of Science and \\ Technology, Wuhan Hubei 430081, China \\ aemail: zll999-9@163.com, bemail: sahara1990 @163.com, bemail: zxl65@163.com
}

Keywords: Effective Prestress Force; Estimation; Dynamical Method

\begin{abstract}
The relationship between natural frequency and prestress-force is built to evaluate effective prestress-force of beams based on the theory. Two corresponding test mode beams are built up to verify the theory, the experiment results show that the natural frequency will decrease along with prestress-force decreasing, which is coincident with the result of theory. As a result, effective prestress-force is predicted by using the measured natural frequencies. Therefore, the research result demonstrate it is feasible to assess the effective prestress-force with dynamical method.
\end{abstract}

\section{Introduction}

Prestress concrete beam had obtained the fast development in the 1930s, and it had been used widely in bridges and building for its remarkable advantage[1][2]. In the 1950s prestress concrete structure was studied in China, subsequently, it was successful put into use on bridges, and prestress concrete structure played very important role in bridge construction in our country[3]. Furthermore, with the development of transportation construction, the improvement of prestress material, construction technique of prestress-force, structural system, and maintenance technology, prestress concrete structure become more widespread[4]. But In the long-term operations, bridges bear moving vehicle load or overload, in additional, the natural disaster and road accident also play a bad role in bridges, if prestress-force had tossed in this progress, the carrying capacity of bridge structure will decrease greatly, and life of bridge structure will reduce greatly[5]. For the prestress concrete bridge, prestress-force loss is one of the most important causes resulting in bridge ruin[6], therefore, estimating the bearing capacity of bridge structure with assessing effective prestress-force is a very important method. Now the effective prestress-force is calculated through counting the prestress-force loss base on the theory during design and construction. There are a lot of uncertain factors which influence the prestress-force loss during operation[7], the actual effective prestress-force is different of theoretical prestress-force loss, which will reduce the safety of the structure[8]. It is very important to look for a new method to assess the effective prestress-force.

It will be very hard to evaluate the effective prestress-force without a preassembled apparatus during construction, we can assess the effective prestress-force through measuring the natural frequency and modal of bridge structure. In this paper, the relationship between natural frequency and prestress-force was obtained on the base of theory, and test verified the availability of this method.

\section{Theory of approach}

Based on the previous study[9], an effective flexural rigidity model of a simply supported PSC beam with an eccentric tendon is schematized as shown in Figure 1. The tendon is initially stretched and anchored to introduce prestressing effect. Then, the structure is in axial compression due to the prestress loads applied at the anchorage edges. That is, the structure is initially deformed in compression and the tendon is still in tension due to the constraint after elastic stretching.

The governing differential equation of the effective flexural rigidity model of the PSC beam with the curved tendon [as shown in Figure 1] is expressed by: 


$$
\begin{aligned}
& \frac{\partial^{2}}{\partial x^{2}}\left(E_{r} I_{r} \frac{\partial^{2} y}{\partial x^{2}}\right)+m_{r} \frac{\partial^{2} y}{\partial t^{2}}=0 \\
& \begin{array}{|l|l|}
\hline & \text { concrete beam } \\
\hline \text { tendon } & \text { L } \\
\hline y
\end{array}
\end{aligned}
$$

Fig.1. Prestressed beam with a tendon

where $E_{r} I_{r}$ is the effective flexural rigidity of PSC beam section which is assumed constant along the entire length of the beam and $m_{r}$ is the effective mass per unit length of the beam. The effective flexural rigidity of PSC beam can be evaluated as the combination of the flexural rigidity of concrete beam section and the equivalent flexural rigidity of tendon. As shown in Figure 1, the effective flexural rigidity $E_{r} I_{r}$ and the effective mass $m_{r}$ of the PSC beam can be estimated, respectively, as follows:

$$
\begin{aligned}
& E_{r} I_{r}=E_{c} I_{c}+E_{p} I_{p} \\
& m_{r}=\rho_{c} A_{c}+\rho_{p} A_{p}
\end{aligned}
$$

Where $E_{c}$ is the elastic modulus of concrete, $I c$ is the second moment of concrete beam's cross-section area, $E_{p}$ is the elastic modulus of steel tendon, and $I_{p}$ is the second moment of tendon's cross-section area. Also, $\rho_{c} A_{c}$ is the concrete mass per unit length and $\rho_{p} A_{p}$ is the tendon mass per unit length.

The equivalent flexural rigidity of tendon is derived from analyzing flexural vibration of tendon of arc-length $L$. By analyzing a pin-pin ended cable with the same span length $L$ and the mass property $\rho_{p} A_{p}$ as the tendon, as shown in Figure 2(a), the cable subjected to tension force $T$ leads the $n^{\text {th }}$ natural frequency $\omega_{n}$, By setting a corresponding beam with a span length $L$ which produces the same $n^{\text {th }}$ natural frequency $\omega_{n}$, as shown in Figure 2(b), the equivalent flexural rigidity $E_{p} I_{p}$ to the tension force $T$ is obtained as:

$$
\begin{aligned}
& \omega_{n}^{2}=\left(\frac{n \pi}{L}\right)^{2} \frac{T}{\rho_{p} A_{p}}=\left(\frac{n \pi}{L}\right)^{4} \frac{E_{p} I_{p}}{\rho_{p} A_{p}} \\
& E_{p} I_{p}=T\left(\frac{L}{n \pi}\right)^{2}
\end{aligned}
$$

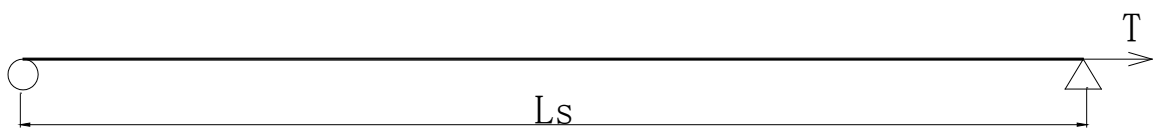

(a) Pin-pin ended cable subjected to tension force $T$

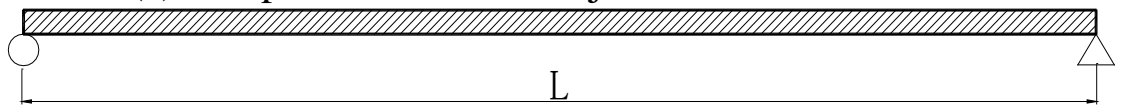

(b) Equivalent beam with flexural rigidity $E_{p} I_{p}$

Fig.2. Flexural rigidity model of tendon subjected to tension force $T$

where $n$ is mode number and $T$ is tension force of cable. On substituting equation 5 into equation 2 and furthermore applying equation 2 with appropriate boundary conditions to equation 1, the $n^{\text {th }}$ natural frequency of the effective flexural rigidity model of the PSC beam can be obtained as:

$\omega_{n}^{2}=\left(\frac{n \pi}{L}\right)^{4} \frac{1}{m_{r}}\left(E_{c} I_{c}+T\left(\frac{L}{n \pi}\right)^{2}\right)$

Once the $n^{\text {th }}$ natural frequency of the PSC beam is known, the prestress force can be identified from an inverse solution of equation 6 , as follows:

$T_{n}=\left(\omega_{n}^{2} m_{r}\left(\frac{L}{n \pi}\right)^{2}-E_{c} I_{c}\left(\frac{n \pi}{L}\right)^{2}\right)$ 


\section{Test Methods}

In order to investigate the variation of natural frequency according to the level of prestress force at the grouted tendon, post-tensioned concrete beams with the grouted tendon type were manufactured. The total number of post-tensioned concrete beams is 2 and their length is $3.2 \mathrm{~m}$ and area is $0.04(0.2 \times 0.2 \mathrm{~m}) \mathrm{m}^{2}$. The beams were simply supported with the span length of $3.0 \mathrm{~m}$, two simple supports of the beams were simulated by using thin rubber pads as interfaces between the beam and the rigid frame. The post-tensioned concrete beam was reinforced longitudinally with a seven-wire straight concentric mono-strand with $15.2 \mathrm{~mm}$ diameter and the stirrups were used for the positioning of the duct for prestressed strand with reinforcing bar of $10 \mathrm{~mm}$ in diameter. The stirrups were used to facilitate the position of the top bars. The tendon was placed in a $25 \mathrm{~mm}$ diameter duct that remained ungrouted. The acceleration occurring from both tests, was measured by means of piezo-electrical sensors. In order to get the various mode shapes, seven accelerometers sensors were placed on top of the beam with a constant $0.5 \mathrm{~m}$ interval. The impact excitation was applied in vertical direction by an hammer at a location $0.75 \mathrm{~m}$ distanced from the right edge. The schematic of the test structure is shown in Figure 3.

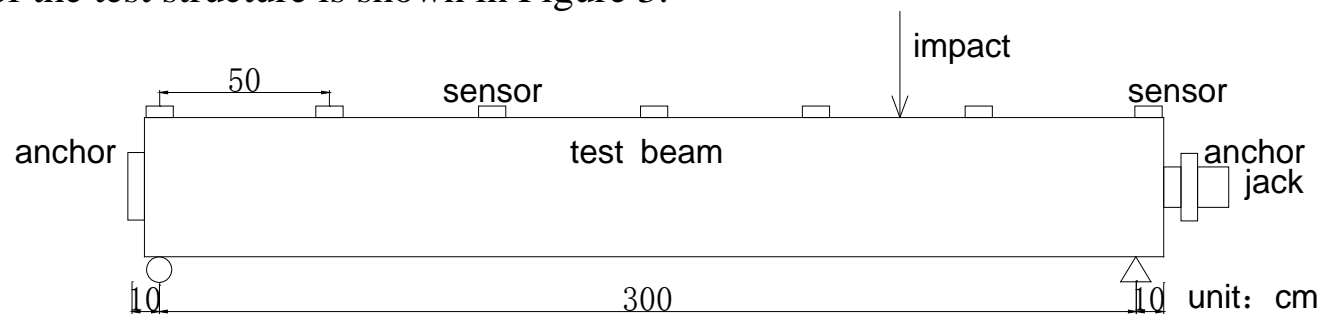

Fig.3. The schematic of the test model

Axial prestress forces were introduced into the tendon by a stressing jack as the tendon was anchored at one end and pulled out at the other. A load cell was installed at the right end to measure accurately the prestress force which was applied to the post-tensioned concrete beam. Each test was conducted after the desired prestress force has been applied and the cable has been anchored. During the measurement, the stressing jack was removed from the beam to avoid the influence of the jack weight on dynamic characteristics of the test structure. The prestress force was applied to the test structure up to seven different prestress cases. The maximum and minimum prestress forces were set to $120 \mathrm{kN}$ and $0 \mathrm{kN}$, respectively. The force was uniformly decreased by $20 \mathrm{kN}$ for each prestress-loss case.

\section{Test results}

For the two prestress cases, natural frequencies of the first mode were extracted as summarized in Table 1. The variation of natural frequencies with respect to the prestress force as the reference are given in Table 1. The corresponding mode shapes of the first two bending modes were extracted as shown in Figure 4. Note that mode shapes were not changed significantly due to the change in prestress forces.

Table 1. Natural frequencies of two test beams

\begin{tabular}{ccccccc}
\hline \multirow{2}{*}{$\begin{array}{c}\text { Prestress } \\
\text { Force } \\
(\mathrm{kN})\end{array}$} & \multicolumn{5}{c}{ NaturalFrequency $(\mathrm{Hz})$} \\
\cline { 2 - 7 } & Experiment & Theory & Error(\%) & Experiment & Theory & Error(\%) \\
\hline 0 & 35.788 & 35.821 & 0.09 & 35.932 & 35.954 & 0.06 \\
20 & 36.083 & 36.113 & 0.08 & 36.241 & 36.43 & 0.52 \\
40 & 36.702 & 36.809 & 0.29 & 36.562 & 36.589 & 0.07 \\
60 & 37.682 & 37.702 & 0.05 & 37.611 & 37.712 & 0.27 \\
80 & 38.553 & 38.679 & 0.33 & 38.268 & 38.311 & 0.11 \\
100 & 39.478 & 39.512 & 0.09 & 39.352 & 39.487 & 0.34 \\
120 & 40.501 & 40.731 & 0.56 & 40.313 & 40.376 & 0.16 \\
\hline
\end{tabular}




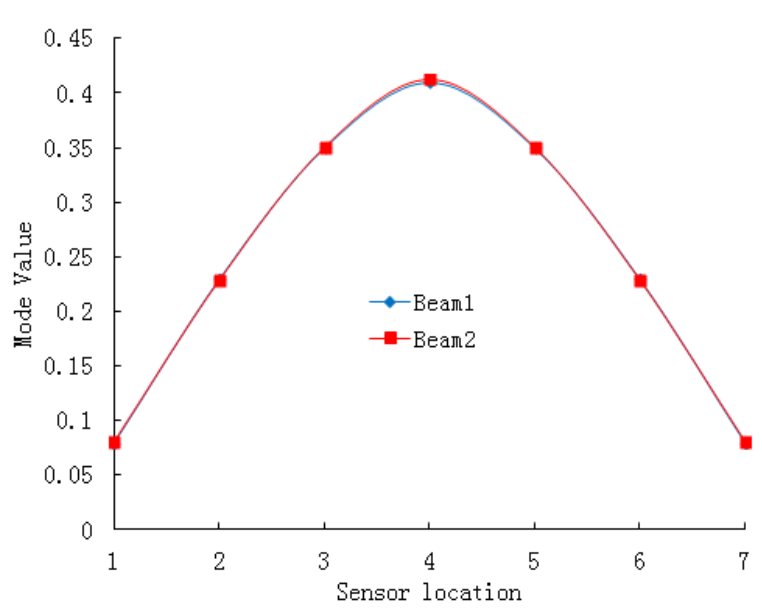

(a) Bending mode shapes of mode 1

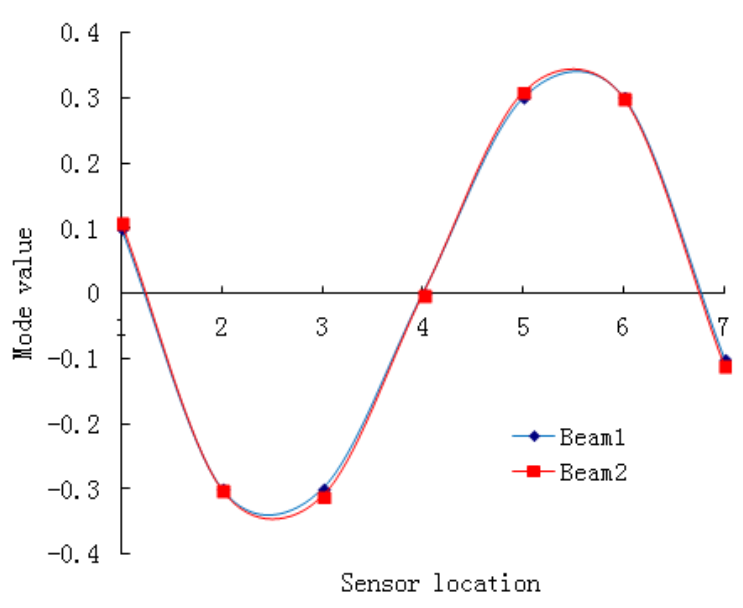

(b) Bending mode shapes of mode 2

Figure 4. Mode shapes from experimental measurement

\section{Summary and Conclusions}

Evaluating effective prestress-force is the key point to ensure bearing capacity of bridge structure. In this paper, the relationship between natural frequency and prestress-force was built based on the theory, and corresponding mode test result shows the natural frequency will decrease along with prestress-force decreasing, which is coincident with the result of theory, therefore, it is feasible to measure natural frequency of bridge structure to assess the effective prestress-force.

\section{Acknowledgement}

In this paper, the research was sponsored by the Talent Project of Hubei Provincal Department of Education (Project No. Q20121109)

\section{References}

[1] Liu Xuewei. Influnce of Vertical Prestressed Steel Anchor Backing Plate installated Dip on Prestressed Loss[D]. Changsha: Hunan University of Science and Technology, 2008.

[2] Fang Zhi, Wang Jian. Vertical Prestressing Loss in the Box Girder of Long-span PC Continuous Bridges[J]. China Civil Engineering Jourual. 2006(7):78-84. .

[3] Du Guanmin, Chen Ai-ping. Control of Anchoring Stress Loss of Vertical Prestressing Bar for Concrete Structure[J]. Bridge Construction, 2006:94-97.

[4] Guo Quan-quan, Li Zhu, Zhang Shan yuan. Study on digital tensioning technique in prestressed concrete structure[J]. China Civil Engineering Journal,2004,37(7):13-17. .

[5] Zhou Mi,He Shuan-hai,Song Yi-fan. Structural evaluation for beams based on deflection testing[J]. Journal of chang'an niversity,2004,24(5):40-42.

[6] Qin Lexun, Cai Jiahang. Stress Loss Control of Vertical Prestress in the Course of Anchoring[J]. Journal of Guangdong Communications Polytechnic, 2007(2):35-37.

[7] Kim, B.H., Jang, J.B., Lee, H.P., 2011b. Longitudinal vibration mechanism of groutedPSC tendon. J. Korean Soc. Civil Eng. 31 (3A), 261 - 267.

[8] Kim, B.H., Jang, J.B., Lee, H.P., 2011a. Feasibility study of estimating prestress forceof grouted tendons. J. Korean Soc. Civil Eng. 30 (2A), 103 - 111.

[9] Kwon, K.S. and Lin, R.M. (2004). "Frequency selection method forFRF-based model updating”, Journal of Sound and Vibration,Vol. 278, No. 1-2, pp. 285-306. 